\title{
Euclidean Proof of the Goldstone Theorem*
}

\author{
K. SymanziK \\ Courant Institute of Mathematical Sciences \\ New York University, New York, New York
}

Received July 17, 1967

\begin{abstract}
The Goldstone theorem in the formulation of Kastreer, RoBInson, and SwIECA is proven in the framework of Euclidean quantum field theory. One utilizes that Schwinger functions have the cluster property in all directions.
\end{abstract}

There is no lack of proofs of the Goldstone theorem [1]. We present here a proof of the theorem as formulated by KASTLER, RoBINSON and SwIEca [2] in the framework of Euclidean quantum field theory [3] derived $[4,5]$ from a Wightman theory [6]. Although such theory requires more assumptions than are known to suffice [2] for the Goldstone theorem, the simplicity of the proof itself is noteworthy and exhibits a feature of the Euclidean approach which is interesting even for Lagrangian-free field theory.

We consider the Wightman theory of a scalar multicomponent field $A(x)$ where we suppress the subscript. Nonscalar fields can be dealt with similarly as below and require merely a straightforward extension of notation. We assume the existence of a local automorphism $A(x) \rightarrow$ $\rightarrow A^{\tau}(x)$ which is generated by a conserved current:

$$
\begin{gathered}
(\partial / \partial \tau) A^{\tau}(x)=i\left[Q_{V}\left(x^{0}\right), A^{\tau}(x)\right] \\
Q_{V}\left(x^{0}\right)=\int_{V} d^{3} x^{\prime} j^{0}\left(x^{0}, \mathbf{x}^{\prime}\right), \quad \mathbf{x} \in V \\
\partial_{\mu} j^{\mu}(x)=0
\end{gathered}
$$

Examples of such automorphisms acting e.g., on the suppressed subscript are one-dimensional subgroups of constant-parameter gauge groups or of internal-symmetry groups. We assume the current operator to be local and to transform as a vector field. We choose a $C^{\infty}$ time-smearing function $f(t)$ with support in $(-T,+T)$ and obeying $\int f(t) d t=1$ and

* Supported by the National Science Foundation. 
rewrite (1), using (2) and commutativity in spacelike distances, as

$$
\begin{aligned}
(\partial / \partial \tau) & \left\langle A^{\tau}\left(x_{1}\right) \ldots A^{\tau}\left(x_{n}\right)\right\rangle=i \int_{|\mathrm{z}| \leqq R} d^{3} z \int_{-T}^{T} f(t) d t \times \\
& \times\left[\left\langle j^{0}\left(R^{\prime}+t, \mathbf{z}\right) A^{\tau}\left(x_{1}\right) \ldots A^{\tau}\left(x_{n}\right)\right\rangle-\right. \\
& \left.-\left\langle A^{\tau}\left(x_{1}\right) \ldots A^{\tau}\left(x_{n}\right) j^{0}\left(-R^{\prime}+t, \mathbf{z}\right)\right\rangle\right]+ \\
& +i \int_{-R^{\prime}}^{R^{\prime}} d z^{0} \int_{|z|=R} d^{2} O_{k}(z) \int_{-T}^{T} f(t) d t\left\langle j^{k}\left(z^{0}+t, \mathbf{z}\right) A^{\tau}\left(x_{1}\right) \ldots A^{\tau}\left(x_{n}\right)\right\rangle
\end{aligned}
$$

where we choose $R$ and $R^{\prime}$ so large that

$$
\operatorname{Max}_{i}\left|x_{i}^{0}\right|<R^{\prime}-T, \quad \operatorname{Max}_{i}\left(2\left|\mathbf{x}_{i}\right|+\left|x_{i}^{0}\right|\right)<R-R^{\prime}-T .
$$

We take (3) as the rigorization of (1).

We now multiply the time arguments in (3) by the real number $\alpha$, taking values in $\left[\frac{1}{2}, 1\right]$. Defining

and

$$
\left\langle A^{\tau}\left(\alpha x_{1}^{0}, \mathbf{x}_{1}\right) \ldots A^{\tau}\left(\alpha x_{n}^{0}, \mathbf{x}_{n}\right)\right\rangle=W_{\alpha}^{\tau}\left(x_{1} \ldots x_{n}\right)
$$

$$
\begin{aligned}
\left\langle\mathrm{j}^{\mu}\left(\alpha z_{n}^{0}, \mathbf{z}\right) A^{\tau}\left(\alpha x_{1}^{0}, \mathbf{z}_{1}\right) \ldots A^{\tau}\left(\alpha x_{n}^{0}, \mathbf{x}_{n}\right)\right\rangle & =W_{\alpha}^{\mu, \tau}\left(z, x_{1} \ldots x_{n}\right), \\
\left\langle A^{\tau}\left(\alpha x_{1}^{0}, \mathbf{x}_{1}\right) \ldots A^{\tau}\left(\alpha x_{n}^{0}, \mathbf{x}_{n}\right) j^{\mu}\left(\alpha z^{0}, \mathbf{z}\right)\right\rangle & =W_{\alpha}^{\mu, \tau}\left(x_{1} \ldots x_{n}, z\right)
\end{aligned}
$$

(3) yields

$$
\begin{aligned}
& (\partial / \partial \tau) W_{\alpha}^{\tau}\left(x_{1} \ldots x_{n}\right)=i \int_{|\mathbf{z}| \leqq R} d^{3} z \int_{-T}^{T} f(t) d t \times \\
& \quad \times\left[W_{\alpha}^{0, \tau}\left(\left(R^{\prime}+t, \mathbf{z}\right), x_{1} \ldots x_{n}\right)-W_{\alpha}^{0, \tau}\left(x_{1} \ldots x_{n},\left(-R^{\prime}+t, \mathbf{z}\right)\right)\right]+ \\
& \quad+i \alpha \int_{-R^{\prime}}^{R^{\prime}} d z^{0} \int_{|\mathbf{z}|=R} d^{2} O_{k}(z) \int_{-T}^{T} f(t) d t W_{\alpha}^{k, \tau}\left(\left(z^{0}+t, \mathbf{z}\right), x_{1} \ldots x_{n}\right)
\end{aligned}
$$

where we have used that the right hand side of (3) is independent of the smearing function $f(t)$. All terms on the right hand side of (4) possess as functions of $\alpha$ analytic continuations into the lower complex half plane $[3,4]$ (for convenience we choose all $x_{i}$ space-like relative to each other, and the time smearing of $z$ serves to remove [7] the singularities connected with timelike distances) and so possesses the left hand side, and under a natural assumption concerning the continuity of the automorphism that continuation is the one under the differentiation sign as seen by integrating over $\tau$ and temporary smearing over the arguments $x_{1} \ldots x_{n}$. The continuations to $\alpha=-i$ are the Schwinger functions

and

$$
W_{-i}^{\tau}\left(x_{1} \ldots x_{n}\right)=S^{\tau}\left(x_{1} \ldots x_{n}\right)
$$

$(+i)^{\delta} \mu_{00} W_{-i}^{\mu, \tau}\left(z, x_{1} \ldots x_{n}\right)=(+i)^{\delta}{ }_{\mu 0} W_{-i}^{\mu, \tau}\left(x_{1} \ldots x_{n}, z\right)=S^{\mu, \tau}\left(z, x_{1} \ldots x_{n}\right)$ 
which are well-defined and real-analytic [4] for any non-coinciding arguments, and Euclidean-covariant such that, e.g.,

$$
S^{\mu, \tau}\left((R z+a),\left(R x_{1}+a\right) \ldots\left(R x_{n}+a\right)\right)=R^{\mu \nu} S^{v, \tau}\left(z, x_{1} \ldots x_{n}\right)
$$

with $R \in S O(4, R)$. (The metric tensor is now the unit tensor while the original metric tensor is $\operatorname{diag}(1,-1,-1,-1)$.) Since due to $(2)$

$$
\partial_{\mu} W^{\mu, \tau}\left(z, x_{1} \ldots x_{n}\right)=0
$$

in particular for purely spacelike separated points, as an analytic relation this entails

$$
\partial_{\mu} S^{\mu, \tau}\left(z, x_{1} \ldots x_{n}\right)=0, \quad z \neq x_{i}, \forall i
$$

at all points of analyticity of the Schwinger function, i.e., when no two points coincide. With (5), (4) may for $\alpha=-i$ be simplified to

$$
(\partial / \partial \tau) S^{\tau}\left(x_{1} \ldots x_{n}\right)=\oint d O^{\mu}(z) S^{\mu, \tau}\left(z, x_{1} \ldots x_{n}\right)
$$

provided the surface, whose normal points outward, encloses all points $x_{i}$.

We now use that Schwinger functions have the strong (i.e., exponential) cluster property $[4,5]$ in all directions if the mass spectrum has a gap between the vacuum and some positive mass ${ }^{1}$. Since $S^{\mu}(0)$ $=(i)^{\delta}{ }_{\mu 0}\left\langle j^{\mu}(0)\right\rangle=0$ due to relativistic invariance, the right hand side of (6) vanishes upon pushing the integration surface to infinity. Therefore, under these circumstances, $S^{\tau}\left(x_{1} \ldots x_{n}\right)$ is independent of $\tau$ and consequently so is $W^{\tau}\left(x_{1} \ldots x_{n}\right)$, wherefrom the implementability of the automorphism by a unitary transformation that leaves the vacuum invariant follows by the reconstruction theorem as in ref. 2. (It is only here that the positive metric of the Hilbert space comes into play.)

We add some remarks. The derivation of (6) can immediately be generalized to yield

$$
\begin{aligned}
\partial^{\mu} S^{\mu, \tau}\left(z, x_{1} \ldots x_{n}\right)= & \sum_{i=1}^{n} \delta\left(z-x_{i}\right) S^{\tau}\left(x_{1} \ldots \underline{x}_{i} \ldots x_{n}\right)+ \\
& + \text { Schwinger terms }
\end{aligned}
$$

where the underlined argument indicates the substitution

$$
A^{\tau}\left(x_{i}\right) \rightarrow(\partial / \partial \tau) A^{\tau}\left(x_{i}\right) \equiv \underline{A}^{\tau}\left(x_{i}\right) .
$$

In (7), Schwinger terms are defined as (because of (5), local) terms that do not contribute in integrations over Euclidean four-space. E.g., we might have

$$
\text { Schwinger terms }=\sum_{i=1}^{n} \square_{z} \delta\left(z-x_{i}\right) S^{\tau}\left(x_{1} \ldots \tilde{x}_{i} \ldots x_{n}\right)
$$

${ }^{1}$ It here suffices that there be such gap in the mass spectrum of the state $\left.j^{\mu}(0)\right\rangle$ for some $\mu$. 
with the tilde denoting a scalar operator ${ }^{2}$ replacement $A^{\tau}\left(x_{i}\right) \rightarrow \widetilde{A}^{\tau}\left(x_{i}\right)$, or

$$
\text { Schwinger terms }=\sum_{i=1}^{n} \partial_{z}^{\mu} \delta\left(z-x_{i}\right) S^{\mu, \tau}\left(x_{1} \ldots \hat{x}_{i} \ldots x_{n}\right)
$$

with a vector operator ${ }^{2}$ replacement $A^{\tau}\left(x_{i}\right) \rightarrow \hat{A}^{\mu, \tau}\left(x_{i}\right)$, etc. The corresponding equal-time commutation relations are

$$
i\left[j^{0}(z), A^{\tau}(x)\right] \delta\left(z^{0}-x^{0}\right)-\underline{A}^{\tau}(x) \delta(z-x)=\Delta_{z} \delta(z-x) \widetilde{A}^{\tau}(x)
$$

respectively

$$
=\partial_{k z} \delta(z-x) \hat{A}^{k, \tau}(x)
$$

where $\Delta_{z}$ is the three-dimensional Laplacian. The "time"-derivative terms that appear in (8) additional to the space-derivative terms from (9) stem from the correction terms [8] at coinciding space-time points by which Green's functions differ from the vacuum expectation values of time-ordered products and Schwinger functions differ from the $\alpha$-continuations, as described before, of such expectation values. These correction terms, which restore covariance, contain $^{3}$ up to $(n-1)^{\text {th }}$ order time derivatives of space-time delta functions if the equal-time commutator contains up to $n^{\text {th }}$ order space derivatives of space delta functions. On the right hand side of (7) also Schwinger terms involving coincidence of three or more arguments could occur.

Similarly as (5) and (6) or, alternatively, (7) were derived, one may derive relations involving the energy-momentum tensor or angularmomentum density tensor upon making suitable assumptions concerning these. In (5) and (6), due to (2) the mentioned fine points concerning the definition of Schwinger functions (as also of Green's functions) at coinciding arguments do not matter. In particular, (5) and (6) and the conclusions drawn therefrom are also valid if the fields are not tempered operator-valued distributions but the more general strictly localizable fields of Jaffe [9] which do occur in some physically relevant models. On the other hand, in the derivation of differential equations for Green's and Schwinger functions as a consequence of field equations $[3,5]$ one encounters "nonconserved currents" and the definition of those functions at coinciding points need be used, as for the derivation of (8) from (9). In the first case, the required information concerning equal-time commutators is the content of the canonical commutation relations which, in turn, depend on the field equations [10].

${ }_{2}$ The vacuum expectation values of $\tilde{A}^{\tau}$ and $\hat{A}^{\mu, \tau}$ must be zero if, as assumed here, $A^{\tau}$ is a scalar field. Of course we tacitly assume that equal-time commutators are meaningful.

3 Of course, one may always add further local correction terms that are covariant by themselves. 


\section{References}

1. Goldstone, J.: Nuovo Cimento 19, 154 (1961).

2. Kastler, D., D. W. Robinson, and A. Swieca: Commun. Math. Phys. 2, 108 (1966).

3. Schwinger, J.: Proc. Nat. Acad. Sci. 44, 956 (1958). - Nakano, T.: Progr. Theor. Phys. (Kyoto) 21, 241 (1959).

4. Ruelle, D.: Thesis (Bruxelles, 1959); Nuovo Cimento 19, 356 (1961).

5. Symanzik, K.: J. Math. Phys. 7, 510 (1966).

6. Wightman, A. S.: Phys. Rev. 101, 860 (1956).

7. Borchers, H. J.: Nuovo Cimento 33, 1600 (1964).

8. E.g., LaM, C. S.: Nuovo Cimento 38, 1755 (1965). - Brown, L. S.: Phys. Rev. 150, 1338 (1966).

9. JAFFE, A.: Phys. Rev. 158, 1454 (1967).

10. Peierls, R. E.: Proc. Roy. Soc. (London) A 214, 143 (1952). - Schwinger, J.: Phys. Rev. 91, 713 (1953). 